Pacific Journal of Mathematics

GROUPS OF HOMEOMORPHISMS OF NORMED LINEAR 


\title{
GROUPS OF HOMEOMORPHISMS OF NORMED LINEAR SPACES
}

\author{
R. A. McCoY
}

\begin{abstract}
For $X$ a Hausdorff space let $H(X)$ be the group of homeomorphisms of $X$. We study here certain subgroups of $H(E)$ where $E$ is an infinite-dimensional normed linear space.
\end{abstract}

The set of homeomorphisms from a topological space $X$ onto itself forms a group $H(X)$ under composition. There are many topologies which can be given to $H(X)$, some of which may make $H(X)$ a topological group. It is natural to ask about the properties of $H(X)$, both algebraic and topological. Also, what relationships are there between $X$ and $H(X)$ ? One way to attack these questions is to study various subgroups of $H(X)$. In this paper we shall investigate certain subgroups of $H(E)$, where $E$ is a normed linear space.

1. Algebraic properties of $H(E)$. Let $X$ be a Hausdorff space. If $A \subset X, S(A)$ will denote the set of elements of $H(X)$ which are supported on $A$. That is, $h \in S(A)$ if and only if $\left.h\right|_{X-A}$ is the identity on $X-A$. Let $\mathscr{B}$ be a base for the topology on $X$. Define $B(X)$ to be the subgroup of $H(X)$ which is generated by those elements of $H(X)$ which are supported on elements of $\mathscr{B}$. Then $h \in B(X)$ if and only if $h=h_{n} \cdots h_{1}$, where for each $i \leqq n, h_{i} \in S\left(B_{i}\right)$ for some $B_{i} \in \mathscr{B}$. A homeomorphism $h \in H(X)$ is said to be stable if $h=$ $h_{n} \cdots h_{1}$, where for each $i \leqq n, h_{i} \in S\left(X-U_{i}\right)$ for some nonempty open set $U_{i}$ in $X$. The stable homeomorphisms of $X, S H(X)$, form a subgroup of $H(X)$.

We shall consider the following possible conditions on $\mathscr{B}$.

B 1. For every $B_{1}, B_{2} \in \mathscr{B}$, there exists an $h \in H(X)$ such that $h\left(B_{1}\right) \subset B_{2}$.

B 1'. For every $B_{1}, B_{2} \in \mathscr{B}$, there exists an $h \in B(X)$ such that $h\left(B_{1}\right) \subset B_{2}$.

B 2. For every $B \in \mathscr{B}$, there exists an $x \in B$ and a pairwise disjoint sequence $\left\{B_{i} \in \mathscr{B} \mid B_{i} \subset B, i=1,2, \cdots\right\}$ which converges to $x$ (i.e., for every open set $U$ containing $x$, there is some $B_{i}$ contained in $U$ ), and there exists an $h \in S(B)$ such that $h\left(B_{i}\right)=B_{i+1}$ for every $i$.

B 3. For every $B \in \mathscr{B}$ and $h \in H(X), h(B) \in \mathscr{B}$.

B 4. For every $B \in \mathscr{B}$, there exists $B^{\prime} \in \mathscr{B}$ such that $B \cup B^{\prime}=$ $X$, and no $B \in \mathscr{B}$ is dense in $X$. 
LEMMA 1.1. If $\mathscr{B}$ satisfies $\mathrm{B} 3$, then $B(X)$ is a normal subgroup of $H(X)$.

Proof. Let $h \in B(X)$ and $f \in H(X)$. Then $h=h_{n} \cdots h_{1}$, where for each $i \leqq n, h_{i} \in S\left(B_{i}\right)$ for some $B_{i} \in \mathscr{B}$. Then

$$
f h f^{-1}=\left(f h_{n} f^{-1}\right) \cdots\left(f h_{1} f^{-1}\right) \cdot
$$

Each $f h_{i} f^{-1} \in S\left(f\left(B_{i}\right)\right)$, so that $f h f^{-1} \in B(X)$.

The following two lemmas can be proved in a manner similar to the proof of Theorem 2 in [9]. Also see [1], [2], and [16].

LEMMA 1.2. Let $\mathscr{B}$ satisfy B1 and B 2, and let $h \in H(X)$ such that $h$ is not the identity. If $f \in B(X)$, then $f$ is a product of conjugates of $h$ and $h^{-1}$ by members of $H(X)$.

LEMMA 1.3. Let $\mathscr{B}$ satisfy B $1^{\prime}$ and B 2, and let $h \in H(X)$ such that $h$ is not the identity. If $f \in B(X)$, then $f$ is a product of conjugates of $h$ and $h^{-1}$ by members of $B(X)$.

THEOREM 1.1. If $\mathscr{B}$ satisfies $\mathrm{B} 1^{\prime}$ and $\mathrm{B} 2$, then $B(X)$ is simple.

Proof. Let $N$ be a normal subgroup of $B(X)$ having more than one element. Let $f \in B(X)$. Choose $h \in N$ such that $h$ is not the identity. Then by Lemma 1.3, $f$ is a product of conjugates of $h$ and $h^{-1}$ by members of $B(X)$. But since $h \in N$ and $N$ is normal in $B(X), f$ is a product of elements of $N$. Therefore $f \in N$, so that $B(X)=N$.

THEOREM 1.2. If $\mathscr{B}$ satisfies $\mathrm{B} 1, \mathrm{~B} 2$, and $\mathrm{B} 3$, then if $B(X)$ is nontrivial, it is the smallest nontrivial normal subgroup of $H(X)$.

Proof. By Lemma 1.1, $B(X)$ is a normal subgroup of $H(X)$. Suppose that $N$ is a normal subgroup of $H(X)$ having more than one element. Let $f \in B(X)$. Choose $h \in N$ such that $h$ is not the identity. Then by Lemma 1.2, $f$ is a porduct of conjugates of $h$ and $h^{-1}$ by members of $H(X)$. But since $h \in N$ and $N$ is normal in $H(X), f$ is a product of elements of $N$. Therefore $f \in N$, so that $B(X) \subset N$.

LEMmA 1.4. If $\mathscr{B}$ satisfies $\mathrm{B} 4$, then $B(X)=S H(X)$.

Proof. Clearly $B(X) \subset S H(X)$. Suppose that $h \in S H(X)$. Then $h=h_{n} \cdots h_{1}$, where for each $i \leqq n, h_{i} \in S\left(X-U_{i}\right)$ for some nonempty open set $U_{i}$ in $X$. Since $\mathscr{B}$ is a base for the topology on $X$, for 
each $i \leqq n$, there is some $B_{i} \in \mathscr{B}$ such that $B_{i} \subset U_{i}$. By property B 4, for each $i \leqq n$, there exists $B_{i}^{\prime} \in \mathscr{B}$ such that $B_{i} \cup B_{i}^{\prime}=X$. Then each $h_{i}$ is an element of $S\left(B_{i}^{\prime}\right)$. Thus $h \in B(X)$.

Theorem 1.1 and Lemma 1.4 then give conditions which imply that $H(X)$ is a simple group.

THEOREM 1.3. If $\mathscr{B}$ satisfies $\mathrm{B} 1^{\prime}, \mathrm{B} 2$, and $\mathrm{B} 4$, and if every element of $H(X)$ is stable, then $H(X)$ is simple.

Now let us consider the special case of the group of homeomorphisms on a normed linear space or a manifold modeled on a normed linear space. $E$ will always denote a normed linear space, and $M$ will be a connected manifold modeled on $E$. By that we mean a connected paracompact space such that every point in $M$ is contained in an open subset of $M$ which is homeomorphic to $E$. If $E$ is finitedimensional it will be permissible to allow $M$ to have boundary.

For finite-dimensional $E$, Fisher defined in [9] a base for $M$ which satisfies B1, B 1', B 2, and B 3. A similar base for $M$ can be found when $E$ is infinite-dimensional.

Lemma 1.5. If $E$ is infinite-dimensional, $M$ has a base $\mathscr{B}$ which satisfies $\mathrm{B} 1, \mathrm{~B} 1^{\prime}, \mathrm{B} 2$, and $\mathrm{B} 3$.

Proof. Take $\mathscr{B}$ to consist of all collared open cells in $M$. By a collared open cell in $M$ is meant the interior of a collared cell in $M$. $C$ is a collared cell in $M$ if there exists a homeomorphism from the triple $\left(B_{2} ; B_{1}, S_{2}\right)$ in $E$ onto the triple $\left(C^{\prime} ; C, B d C^{\prime}\right)$ in $M$, where $C^{\prime}$ is some subset of $M$, where $B_{r}=\{x \in E \mid\|x\| \leqq r\}$, and where $S_{r}=B d B_{r}$.

Property B 1 follows from B 1', and B 3 follows from the definition of $\mathscr{B}$. We shall outline the proof that $\mathscr{B}$ satisfies $B 1^{\prime}$ and $\mathrm{B} 2$ by using a similar technique to that which was used in [9]. Let $Q_{1}, Q_{2} \in \mathscr{B}$. Since $M$ is connected, there are a finite number of elements of $\mathscr{B}$, say $Q^{1}, \cdots, Q^{n}$, such that $Q^{1}=Q_{1}, Q^{n}=Q_{2}$, and $Q^{i} \cap Q^{i+1} \neq \varnothing$ for $i<n$. For each $i<n$, let $f_{i}$ be a homeomorphism from $\left(B_{2} ; B_{1}, S_{2}\right)$ onto $\left(C_{i} ; C l Q^{i}, B d C_{i}\right)$, where $C_{i}$ is some subset of $M$. Also for each $i<n$, we can define a $g_{i} \in S\left(B_{3 / 2}\right)$ such that

$$
g_{i}\left(B_{1}\right) \subset f_{i}^{-1}\left(Q^{i} \cap Q^{i+1}\right) \text {. }
$$

Then define $h=f_{n-1} g_{n-1} f_{n-1}^{-1} \cdots f_{1} g_{1} f_{1}^{-1}$. Since for each $i<n$, $f_{i}\left(\right.$ Int $\left.B_{3 / 2}\right) \in \mathscr{B}$, then $h \in B(M)$. Also $h\left(Q_{1}\right) \subset Q_{2}$.

To establish that $\mathscr{B}$ satisfies $\mathrm{B} 2$, let $Q \in \mathscr{B}$. Let $f$ be a homeomorphism from $\left(B_{2} ; B_{1}, S_{2}\right)$ onto $(C l ; C Q, B d C)$ for some set $C$ in $M$. 
Define $g \in H\left(B_{2}\right)$ by $g(y)=\|y\| y$ for $y \in B_{1}$, and $g(y)=y$ for $y \in B_{2}-B_{1}$. Let $x=f(0)$, and choose $z \in S_{3 / 8}$. For each positive integer $i$, set $Q_{i}=f g^{i}\left(\operatorname{Int} B_{1 / 9}(z)\right)$. Then define $h \in S(Q)$ by $h(y)=$ $f g f^{-1}(y)$ if $y \in C$, and $h(y)=y$ if $y \in M-C$. It can be verified that the sequence $\left\{Q_{i}\right\}$ is pairwise disjoint and converges to $x$, and that $h\left(Q_{i}\right)=Q_{i+1}$ for every $i$.

LEMma 1.6. If $E$ is infinite-dimensional, it has a base $\mathscr{B}$ which satisfies B1, B 1', B 2, B 3, and B 4 .

Proof. As in Lemma 1.5, take $\mathscr{B}$ to consist of all collared open cells in $E$. Hence $\mathscr{B}$ satisfies B1, B 1', B2, and B 3. Klee showed in [13] that if $E$ is infinite-dimensional, there is a $\varphi \in H(E)$ such that $\varphi\left(B_{1}\right)=E-$ Int $B_{1}$. Therefore complements of collared cells are collared open cells. Then to see that $\mathscr{B}$ satisfies B 4, let $Q \in \mathscr{B}$. From Theorem 4.1 in [14] it is seen that $Q$ is tame, so that there exists an $f \in H(E)$ such that $f(Q)=\operatorname{Int} B_{1}$. Let $Q^{\prime}=E-f^{-1}\left(B_{1 / 2}\right)$, which is thus in $\mathscr{B}$ because of Klee's result. Clearly $Q \cup Q^{\prime}=E$.

The next two theorems then follow from Theorem 1.1, Theorem 1.2, Lemma 1.4, Lemma 1.5 and Lemma 1.6.

THeOREM 1.4. $M$ has a base $\mathscr{B}$ such that $B(M)$ is the smallest nontrivial normal subgroup of $H(M)$ and is simple.

Theorem 1.5. If $E$ is infinite-dimentional, then $S H(E)$ is the smallest nontrivial normal subgroup of $H(E)$ and is simple.

It was shown in [8] that if $E$ is homeomorphic to the countably infinite product of copies of itself (we shall abreviate this statement as $E \sim E^{\omega}$ ), then $S H(E)=H(E)$.

THeorem 1.6. If $E \sim E^{\omega}$, then $H(E)$ is simple.

It should be noted that if $E$ is an infinite-dimensional Hilbert space, then $E \sim E^{\omega}$ [5]. Also, all reflexive Banach spaces are homeomorphic to Hilbert spaces [6]. In fact, at this time there seems to be no known infinite-dimensional $E$ which is not homeomorphic to $E^{\omega}$.

2. Stable structure on $E$. Whittaker defines the following terms in [18]. Let $\mathscr{K}(X)$ be the set of nonempty connected open subsets $U$ of $X$ such that for every $x, y \in U$, there exists an $f \in S(U)$ with $f(x)=y$. Set $K(X)=\cup \mathscr{K}(X)$, which is an open subset of $X$. 
Finally, define $R(X)$ to be the set of $h \in H(X)$ such that for every $x \in K(X)$ and every connected open subset $U$ of $K(X)$ containing $x$ and $h(x)$, there is a neighborhood $V$ of $x$ and an $f \in S(U)$ satisfying $\left.f\right|_{V}=\left.h\right|_{V}$.

It was shown in [18] that if $X$ is a Hausdorff space such that each open subset contains a member of $\mathscr{K}(X)$, and $K(X)$ cannot be separated by any two points, then $R(X)$ is a normal subgroup of $H(X)$.

As in the previous section, $E$ will denote a normed linear space, and $M$ will be a connected manifold modeled on $E$.

Lemma 2.1. $\mathscr{K}(M)$ is a base for the topology on $M$, and $K(M)=M$.

Proof. If $z \in M$, then there exists a collared open cell $Q$ in $M$ containing $z$. Let $g$ be a homeomorphism from $\left(B_{2} ; B_{1}, S_{2}\right)$ onto $(C$; $C l Q, B d C$ ), for some set $C$ in $M$ (see the proof of Lemma 1.5 for terminology). Let $x, y \in Q$, and set $a=g^{-1}(x)$ and $b=g^{-1}(y)$. Define $h \in H\left(B_{1}\right)$ as follows. First define $h(a)=b$. Next let $c \in B_{1}-\{a\}$. Let $\left\{c^{\prime}\right\}=$ Ray $[a: c] \cap S_{1}$, where Ray $[a: c]$ is the infinite ray from a through $c$. Then $c=a+\alpha\left(c^{\prime}-a\right)$ for some $0<\alpha \leqq 1$. Define $h(c)=b+\alpha\left(c^{\prime}-b\right)$. With $h$ thus defined, define $f \in H(M)$ by $f(\omega)=$ $g h g^{-1}(\omega)$ if $\omega \in Q$, and $f(\omega)=\omega$ if $\omega \in M-Q$. Then $f \in S(Q)$ and $f(x)=y$. Therefore $Q \in \mathscr{K}(M)$, which makes $\mathscr{K}(M)$ a base for the topology on $M$. Then obviously $K(M)=M$.

THEOREM 2.1. If the dimension of $E$ is greater than one, then $R(M)$ is a normal subgroup of $H(M)$.

It was also shown in [18] that $M$ has a stable structure if and only if $R(M)$ does not consist only of the identity on $M$. The concept of a stable structure was introduced and studied in [7]. $M$ has a stable structure if $M=\cup\left\{U_{\alpha} \mid \alpha \in A\right\}$, where the $U_{\alpha}$ are the images of homeomorphisms $h_{\alpha}$ from $B_{1}$ in $E$ into $M$ which satisfy the condition that if $U_{\alpha} \cap U_{\beta} \neq \varnothing$ and $x \in h^{-1}\left(U_{\alpha} \cap U_{\beta}\right)$, then there is a neighborhood $V$ of $x$ and an $f \in S\left(B_{1}\right)$ such that $\left.f\right|_{V}=\left.h_{\beta}^{-1} h_{\alpha}\right|_{V}$. In the next theorem we shall see that for a large class of spaces $E, R(M)$ is all of $H(M)$.

Theorem 2.2. If $E \sim E^{\omega}$, then $R(M)=H(M)$.

Proof. Let $h \in H(M)$. By Lemma 2.1, $K(M)=M$. So let $x \in M$, and let $U$ be a connected open subset of $M$ containing $x$ and $h(x)$. 
Since $E \sim E^{\omega}$, by a result of Henderson and Schori in [10], there exists a homeomorphism $\varphi$ from $M$ into $E$ such that $\varphi(M)$ is open in $E$. Since $\varphi(U)$ is connected, there is a piecewise linear arc, $\alpha$, joining $\varphi(x)$ and $\varphi h(x)$, such that $\alpha \subset \varphi(U)$. By taking an appropriate $\varepsilon$-neighborhood of $\alpha$, a collared cell $C$ can be found contained in $\varphi(U)$ and containing $\alpha$ in its interior. Choose $\delta>0$ such that

$$
B_{\hat{o}}(\varphi h(x)) \subset \operatorname{Int} C \text {. }
$$

Then choose $\varepsilon>0$ such that $B_{\varepsilon}(\varphi(x)) \subset \varphi h^{-1} \varphi^{-1}\left(\operatorname{Int} B_{j}(\varphi h(x))\right) \cap \operatorname{Int} C$. In [8] it is shown that $S H(E)=H(E)$ if and only if the strong annulus conjecture for $E$ is true. Then since $S H(E)=H(E)$ for $E$ such that $E \sim E^{\omega}$, we may apply the strong annulus conjecture here. Thus there exists $g \in S(C)$ such that $\left.g\right|_{B_{\varepsilon}(\varphi(x))}=\left.\varphi h \varphi^{-1}\right|_{B_{\varepsilon}(\varphi(x))}$. Define $f \in S(U)$ by $f=\varphi^{-1} g \varphi$ and let $V=\varphi^{-1}\left(\operatorname{Int} B_{\varepsilon}(\varphi(x))\right)$. Then $\left.f\right|_{V}=\left.h\right|_{V}$ as desired, so that $h \in R(M)$.

CoRollary. If $E \sim E^{\omega}$, then $M$ has a stable structure.

3. Topological propeties of $H(E)$. Let $X$ be a Hausdorff space, and let $\mathscr{C}$ be a collection of closed subsets of $X$. Define $H_{\mathscr{E}}(X)$ to be $H(X)$ along with the topology generated by the collection

$$
\{[C, U] \mid C \in \mathscr{C} \text { and } U \text { is open in } X\},
$$

where

$$
[C, U]=\{h \in H(X) \mid h(C) \subset U\} .
$$

$X$ is (stably) $\mathscr{C}$-homogeneous if every homeomorphism between elements of $\mathscr{C}$ can be extended to a (stable) homeomorphism in $H(X)$.

For the remainder of this section, $F$ will be a locally convex, linear topological space such that $F \sim F \times F$. If $A$ is a closed subset of $F$, then $A$ is $F$-deficient if there exists a homeomorphism $h$ from $F$ onto $F \times F$ such that $h(A) \subset F \times\{0\}$. It is a standard technique (see [12] and [4]) that $F$ is stably $\mathscr{C}$-homogeneous if $\mathscr{C}$ has the property that for $C, D \in \mathscr{C}, C \cup D$ is $F$-deficient. Lemma 3.1 is a partial converse to this. In Lemma 3.1, Theorem 3.1, and Theorem 3.2, we shall take $\mathscr{C}$ to be closed under finite unions and under homeomorphisms (i.e., if $C, D \in \mathscr{C}$, then $C \cup D \in \mathscr{C}$; and if $C \in \mathscr{C}$, then $h(C) \in \mathscr{C}$ for every $h \in H(F)$ ).

Lemma 3.1. If $F$ is $\mathscr{C}$-homogeneous, then every element of $\mathscr{C}$ is F-deficient.

Proof. Let $C \in \mathscr{C}$, and let $f$ be a homeomorphism from $F$ onto 
$F \times F$. Then the homeomorphism from $C$ onto $f^{-1}(C \times\{0\})$ can be extended to some $g \in H(F)$. Let $h=f g$, so that

$$
h(C)=f g(C)=f f^{-1}(C \times\{0\})=C \times\{0\} \subset F \times\{0\} .
$$

THEOREM 3.1. If $F$ is C-homogeneous, then it is stably CO-homogeneous.

THeOREM 3.2. Let $F$ be C C-homogeneous. Then $S H(F)=H(F)$ if and only if $S H_{\mathscr{E}}(F)$ is open in $H_{\mathscr{E}}(F)$.

Proof. Suppose $S H_{\mathscr{E}}(F)$ is open in $H_{\mathscr{E}}(F)$, and let $h \in H(F)$. Let $\bigcap_{i=1}^{n}\left[C_{i}, U_{i}\right]$ be a neighborhood of the identity on $F$ which is contained in $S H(F)$, where $C_{i} \in \mathscr{C}$ and $U_{i}$ is open for $i \leqq n$. By Theorem 3.1, there exists a $g \in S H(F)$ such that $\left.g\right|_{\cup_{i=1}^{n} C_{i}}=\left.h\right|_{\cup_{i=1}^{n} C_{i}}$. Then $g^{-1} h\left(C_{i}\right) \subset U_{i}$ for $i \leqq n$, so that $g^{-1} h \in S H(F)$. Therefore $h=$ $g\left(g^{-1} h\right) \in S H(F)$.

The following corollary to Theorem 3.2 then is true because infinite-dimensional Fréchet spaces are homogeneous with respect to compact sets, which in turn follows from Michael's version of the Bartle-Graves Theorem, found for example in [15], and from the fact that separable infinite-dimensional Fréchet spaces are homeomorphic to separable Hilbert space, which can be found in [3].

Corollary. Let $F$ be a Fréchet space such that $F \sim F \times F$. Then $S H(F)=H(F)$ if and only if $S H(F)$ is open in $H(F)$ under the compact-open topology.

Kirby showed in [11] that if $E$ is finite-dimensional, then $S H(E)$ is open in $H(E)$ under the compact-open topology. But he made use of the fact that $H(E)$ with the compact-open topology forms a topological group. This is not the case for infinite-dimensional $E$. We might ask the following questions. If $H_{\mathscr{G}}(E)$ is a topological group, is $S H_{\mathscr{E}}(E)$ open in $H_{\mathscr{E}}(E)$ ? Which classes, $\mathscr{C}$, make $H_{\mathscr{E}}(E)$ into a topological group? One answer to this last question is the following theorem.

THEOREM 3.3. Let $E$ be an infinite-dimensional normed linear space, and let $M$ be a connected manifold modeled on $E$. If $\mathscr{C}$ consists of the collared cells in $E$ or $M$, respectively, then $H_{\mathscr{G}}(E)$ is a topological group and $H_{\mathscr{E}}(M)$ is a topological semigroup. If $\mathscr{C}$ consists of the collared cells in $M$ and the complements of the interiors of the collared cells in $M$, then $H_{\mathscr{E}}(M)$ is a topological group. 
Proof. Let $h_{1}, h_{2} \in H(E)$ (or $H(M)$ ). Let $\bigcap_{i=1}^{n}\left[B_{i}, U_{i}\right]$ be an open set in $H(E)$ containing $h_{2} h_{1}$, where each $B_{i} \in \mathscr{C}$. For each $i \leqq n$, let $C_{i} \in \mathscr{C}$ which is contained in $h_{2}^{-1}\left(U_{i}\right)$, such that $h_{1}\left(B_{i}\right) \subset \operatorname{Int} C_{i}$. Such a $C_{i}$ can be found since a collared cell is collared in every open set containing it [17]. Then $h_{2}\left(C_{i}\right) \subset U_{i}$. Let $g_{1} \in \bigcap_{i=1}^{n}\left[B_{i}\right.$, Int $\left.C_{i}\right]$ and $g_{2} \in \bigcap_{i=1}^{n}\left[C_{i}, U_{i}\right]$. Then $g_{2} g_{1}\left(B_{i}\right) \subset g_{2}\left(\operatorname{Int} C_{i}^{\prime}\right) \subset U_{i}$.

Let $h \in H(E)$ (or $H(M)$ ). Let $\bigcap_{i=1}^{n}\left[B_{i}, U_{i}\right]$ be an open set in $H(E)$ containing $h^{-1}$, where each $B_{i} \in \mathscr{C}$. For each $i \leqq n$, let $D_{i} \in \mathscr{C}$ which is contained in $U_{i}$, such that $h^{-1}\left(B_{i}\right) \subset \operatorname{Int} D_{i}$. Let $C_{i}=$ $E$-Int $D_{i}$ which is an element of $\mathscr{C}$ (see the proof of Lemma 1.6). Then $\quad h\left(C_{i}\right)=h\left(E-\operatorname{Int} D_{i}\right) \subset h\left(E-h^{-1}\left(B_{i}\right)\right)=E-B_{i}$. Let $g \in \bigcap_{i=1}^{n}\left[C_{i}, E-B_{i}\right]$. Then $C_{i} \subset g^{-1}\left(E-B_{i}\right)=E-g^{-1}\left(B_{i}\right)$, so that $g^{-1}\left(B_{i}\right) \subset E-C_{i}=\operatorname{Int} D_{i} \subset U_{i}$.

\section{REFERENCES}

1. R. D. Anderson, The algebraic simplicity of certain groups of homeomorphisms, Amer. J. Math., 80 (1958), 955-963.

2. - On homeomorphisms as products of conjugates of a given homeomorphism and its inverse, Topology of 3-manifolds, 1962.

3. - Hilbert space is homeomorphic to the countable infinite product of lines, Bull. Amer. Math. Soc., 72 (1966), 515-519.

4. - Topological properties of the Hilbert cube and the infinite product of open intervals, Trans. Amer. Math. Soc., 126 (1967), 200-216.

5. C. Bessaga, On topological classification of complete linear metric spaces, Fundamenta Mathematicae, 56 (1965), 251-288.

6. — Topological equivalence of non-separable reflexive Banach spaces. Ordinal resolutions of identity and monotone basis, Bull. Acad. Polon. Sci., Ser. Sci. Math., Astr. et Phys., 15 (1967), 397-399.

7. M. Brown and H. Gluck, Stable structures on manifolds. I: Homeomorphisms of $S^{n}$, Ann. of Math., (2) $\mathbf{7 9}$ (1964), 1-17.

8. D. Curtis and R. A. McCoy, Stable homeomorphisms on infinite-dimensional normed linear spaces, Proc. Amer. Math. Soc., 28 (1971), 496-500.

9. G. M. Fisher, On the group of all homeomorphisms of a manifold, Trans. Amer. Math. Soc., 97 (1960), 193-212.

10. D. W. Henderson and R. Schori, Topological classification of in fininite dimensional manifolds by homotopy type, Bull. Amer. Math. Soc., 76 (1970), 121-124.

11. R. C. Kirby, Stable homeomorphisms and the annulus conjecture, Ann. of Math., 89 (1969), 575-582.

12. V. L. Klee, Some topological properties of convex sets, Trans. Amer. Math. Soc., 78 (1955), 30-45.

13. - A note on topological properties of normed linear spaces, Proc. Amer. Math. Soc., 7 (1956), 673-674.

14. R. A. McCoy, Cells and cellularity in infinite-dimensional normed linear spaces, (to appear).

15. E. A. Michael, Continuous selections I, Ann. of Math., 63 (1956), 361-382.

16. E. Nunnally, Dilations on invertible spaces, Trans. Amer. Math. Soc., 123 (1966), 437-448.

17. D. E. Sanderson, An infinite-dimensional Schoenfliess Theorem, Trans. Amer. Math. Soc., 148 (1970), 33-40. 
18. J. V. Whittaker, Some normal subgroups of homeomorphisms, Trans. Amer. Math. Soc., 123 (1966), 88-98.

Received June 16, 1970 and in revised form January 11, 1971.

Virginia Polytechnic Institute

AND

State University 



\title{
PACIFIC JOURNAL OF MATHEMATICS
}

\author{
EDITORS
}

\author{
H. SAMELSON \\ Stanford University \\ Stanford, California 94305 \\ C. R. HOBBY \\ University of Washington \\ Seattle, Washington 98105
}

J. DugundJI

Department of Mathematics

University of Southern California

Los Angeles, California 90007

RICHARD ARENS

University of California

Los Angeles, California 90024

\section{ASSOCIATE EDITORS}
E. F. BeCKenBaCH
B. H. NeUMANN
F. WOLF
K. Yoshida

\section{SUPPORTING INSTITUTIONS}

\author{
UNIVERSITY OF BRITISH COLUMBIA \\ CALIFORNIA INSTITUTE OF TECHNOLOGY \\ UNIVERSITY OF CALIFORNIA \\ MONTANA STATE UNIVERSITY \\ UNIVERSITY OF NEVADA \\ NEW MEXICO STATE UNIVERSITY \\ OREGON STATE UNIVERSITY \\ UNIVERSITY OF OREGON \\ OSAKA UNIVERSITY
}

\author{
UNIVERSITY OF SOUTHERN CALIFORNIA \\ STANFORD UNIVERSITY \\ UNIVERSITY OF TOKYO \\ UNIVERSITY OF UTAH \\ WASHINGTON STATE UNIVERSITY \\ UNIVERSITY OF WASHINGTON \\ AMERICAN MATHEMATICAL SOCIETY \\ NAVAL WEAPONS CENTER
}

The Supporting Institutions listed above contribute to the cost of publication of this Journal, but they are not owners or publishers and have no responsibility for its content or policies.

Mathematical papers intended for publication in the Pacific Journal of Mathematics should be in typed form or offset-reproduced, (not dittoed), double spaced with large margins. Underline Greek letters in red, German in green, and script in blue. The first paragraph or two must be capable of being used separately as a synopsis of the entire paper. The editorial "we" must not be used in the synopsis, and items of the bibliography should not be cited there unless absolutely necessary, in which case they must be identified by author and Journal, rather than by item number. Manuscripts, in duplicate if possible, may be sent to any one of the four editors. Please classify according to the scheme of Math. Rev. Index to Vol. 39. All other communications to the editors should be addressed to the managing editor, Richard Arens, University of California, Los Angeles, California, 90024.

50 reprints are provided free for each article; additional copies may be obtained at cost in multiples of 50 .

The Pacific Journal of Mathematics is published monthly. Effective with Volume 16 the price per volume (3 numbers) is $\$ 8.00$; single issues, $\$ 3.00$. Special price for current issues to individual faculty members of supporting institutions and to individual members of the American Mathematical Society: $\$ 4.00$ per volume; single issues $\$ 1.50$. Back numbers are available.

Subscriptions, orders for back numbers, and changes of address should be sent to Pacific Journal of Mathematics, 103 Highland Boulevard, Berkeley, California, 94708.

PUBLISHED BY PACIFIC JOURNAL OF MATHEMATICS, A NON-PROFIT CORPORATION

Printed at Kokusai Bunken Insatsusha (International Academic Printing Co., Ltd.), 270, 3chome Totsuka-cho, Shinjuku-ku, Tokyo 160, Japan. 


\section{Pacific Journal of Mathematics}

\section{Vol. 39, No. $3 \quad$ July, 1971}

William O'Bannon Alltop, 5-designs in affine spaces ................... 547

B. G. Basmaji, Real-valued characters of metacyclic groups ................. 553

Miroslav Benda, On saturated reduced products....................... 557

J. T. Borrego, Haskell Cohen and Esmond Ernest Devun, Uniquely representable semigroups. II.......................................

George Lee Cain Jr. and Mohammed Zuhair Zaki Nashed, Fixed points and stability for a sum of two operators in locally convex spaces ....................

Donald Richard Chalice, Restrictions of Banach function spaces ...............

Eugene Frank Cornelius, Jr., A generalization of separable groups ..............

Joel L. Cunningham, Primes in products of rings ......................

Robert Alan Morris, On the Brauer group of $Z$.

593

603

615

David Earl Dobbs, Amitsur cohomology of algebraic number rings ...............

Charles F. Dunkl and Donald Edward Ramirez, Fourier-Stieltjes transforms and

weakly almost periodic functionals for compact groups ...................

Hicham Fakhoury, Structures uniformes faibles sur une classe de cônes et

d'ensembles convexes ......................................

Leslie R. Fletcher, A note on C $\theta \theta$-groups.

Humphrey Sek-Ching Fong and Louis Sucheston, On the ratio ergodic theorem for

semi-groups............................................

James Arthur Gerhard, Subdirectly irreducible idempotent semigroups ...........

Thomas Eric Hall, Orthodox semigroups.....................

Marcel Herzog, $C \theta \theta$-groups involving no Suzuki groups ..........

669

687

John Walter Hinrichsen, Concerning web-like continua ..........

691

Frank Norris Huggins, A generalization of a theorem of F. Riesz.

695

Carlos Johnson, Jr., On certain poset and semilattice homomorphisms

703

Alan Leslie Lambert, Strictly cyclic operator algebras ...........

717

Howard Wilson Lambert, Planar surfaces in knot manifolds . . .

727

Robert Allen McCoy, Groups of homeomorphisms of normed linear spaces ....... 735

T. S. Nanjundiah, Refinements of Wallis's estimate and their generalizations ...... 745

Roger David Nussbaum, A geometric approach to the fixed point index .......... 751

John Emanuel de Pillis, Convexity properties of a generalized numerical range .... 767

Donald C. Ramsey, Generating monomials for finite semigroups ....

783

William T. Reid, A disconjugacy criterion for higher order linear vector differential equations...

Roger Allen Wiegand, Modules over universal regular rings...

Kung-Wei Yang, Compact functors in categories of non-archimedean Banach

spaces.

R. Grant Woods, Correction to: "Co-absolutes of remainders of Stone-Čech compactifications".

Ronald Owen Fulp, Correction to: "Tensor and torsion products of

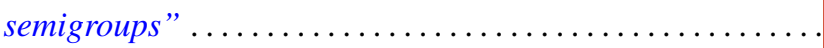

Bruce Alan Barnes, Correction to: "Banach algebras which are ideals in a banach 